doi: $10.2306 /$ scienceasia1513-1874.2012.38.394

\title{
The combined relaxation and non-relativistic approximation of the drift-diffusion model
}

\author{
Jianwei Yang ${ }^{\mathrm{a}, *}$, Xiaoxia Yang ${ }^{\mathrm{b}}$ \\ a College of Mathematics and Information Science, \\ North China University of Water Resources and Electric Power, Zhengzhou 450011, China \\ b The Pilot College of Beijing University of Technology, Beijing 101101, China
}

*Corresponding author, e-mail: yangjianwei@ncwu.edu.cn

Received 15 Mar 2012

Accepted 30 Oct 2012

\begin{abstract}
In this article, we study the combined relaxation and non-relativistic limit for compressible Euler-Maxwell equations for plasma. For the initial data in equilibrium, the convergences of solutions of compressible Euler-Maxwell equations to the solutions of drift-diffusion model are justified rigorously.
\end{abstract}

KEYWORDS: Euler-Maxwell equations, asymptotic limit

\section{INTRODUCTION}

Recently, much attention has been paid to the study of the non-relativistic limit or the short relaxation limit. In this regime, a complicated system may asymptotically be replaced by a much simpler hyperbolic or parabolic system, the behaviour of the latter is either already well understood or easier to analyse. This work concerns the one fluid version of Euler-Maxwell system for semiconductors or plasmas ${ }^{1,2}$ :

$$
\begin{array}{r}
\partial_{t} n+\operatorname{div}(n u)=0, \\
\partial_{t}(n u)+\operatorname{div}(n u \otimes u)+\nabla p(n) \\
=-n(E+\gamma u \times B)-\frac{n u}{\tau}, \\
\epsilon \gamma \partial_{t} E-\nabla \times B=\gamma n u, \gamma \partial_{t} B+\nabla \times E=0,(3) \\
\epsilon \operatorname{div} E=b(x)-n, \quad \operatorname{div} B=0,(4)
\end{array}
$$

with the initial conditions which are periodic in $x$ :

$$
(n, u, E, B)(x, 0)=\left(n_{0}, u_{0}, E_{0}, B_{0}\right) .
$$

The unknown functions are: $n$, the density of electrons; $u \in \mathbb{R}^{3}$, the average velocity of the electrons; $E \in \mathbb{R}^{3}$, the electric field; $B \in \mathbb{R}^{3}$ the magnetic field. They are functions of a 3-dimensional position vector $x \in \mathcal{T}^{3}$ and of the time $t>0$, where $\mathcal{T}^{3}=(\mathbb{R} / 2 \pi \mathbb{Z})^{3}$ is the 3 -dimensional torus. $b(x)$ is the uniform density of ions, and the electron gas is supposed to be barotropic, the pressure being given by $p(n)$ which is supposed to be smooth and strictly increasing for $n>0$. Then for smooth solutions of the Euler-Maxwell system (1)-(5) with $n>0$, equation
(2) is equivalent to

$$
\partial_{t} u+(u \cdot \nabla) u+\nabla h(n)=-E-\gamma u \times B-\frac{u}{\tau},
$$

where the enthalpy $h(n)$ is defined by

$$
h^{\prime}(n)=\frac{p^{\prime}(n)}{n} .
$$

The dimensionless parameters $\epsilon>0, \gamma>0$ and $\tau>0$ can be chosen independently on each other, according to the desired scaling. Physically, $\epsilon$ stands for the scaled Debye length which is related to the Coulomb screening of the charged particles. $\tau$ stands for the momentum relaxation time which describes in a very rough manner the damping effect of the lattice on the charge carriers. And $\gamma$ can be chosen to be proportional $\frac{1}{c}$ where $c=\left(\epsilon_{0} \nu_{0}\right)^{-\frac{1}{2}}$ is the speed of light, with $\epsilon_{0}$ and $\nu_{0}$ being the vacuum permittivity and permeability. These parameters are small compared to the physical size of the known variables. Thus regarding $\epsilon, \gamma$ and $\tau$ as singular perturbation parameters, we can study the limits in the system (1)-(4) as these parameters tend to zero. The limit $\epsilon \rightarrow 0$ leads to $n=b(x)$, which is the quasi-neutrality of the plasma. Hence $\epsilon \rightarrow 0$ is called the quasi-neutral limit. Also, $\tau \rightarrow 0$ and $\gamma \rightarrow 0$ are physically called the zero- relaxation limit and the non-relativistic limit, respectively.

First, let us recall a few results related to (1)(4). There have been numerical simulations ${ }^{3,4}$, but the only rigorous study appears to be that made by Chen etc. in Ref. 5, where a global weak solution is 
proved in one spatial dimension by using compensated compactness. In Ref. 6, Jerome establish a local smooth solution theory for the Cauchy problem of compressible Hydrodynamic-Maxwell systems by the modification of the classical semigroup-resolvent approach of Kato. The convergence of one-fluid (isentropic) Euler-Maxwell system to compressible EulerPoisson system was proven via the non-relativistic limit $^{7}$. The cases of two-fluid and non-isentropic are studied by Yang and Wang in Refs. 8,9. The paper ${ }^{10}$ proved that the combined non-relativistic and quasineutral limit of the (isentropic) Euler-Maxwell is the incompressible Euler equations. We also refer to Refs. 11,12 and Refs. 13, 14 where the Euler equations and Euler-Poisson system are studied, respectively.

To the author's best knowledge, there is no result on the combined non-relativistic and relaxation limit of the above model. In this article we are interested in the non-relativistic regime and small relaxationtime analysis under the conditions $\epsilon=O(1)$. In the following, we are going to motivate the above combined limit. It is known that the relaxation limit $\tau \rightarrow 0$-for fixed $\gamma$-in the Euler-Maxwell model gives the so-called drift diffusion model for plasmas or semiconductors ${ }^{15}$. Likewise, it is known that the relaxation limit $\gamma \rightarrow 0$-for fixed $\tau$-in the EulerMaxwell model gives the Euler-Poisson model ${ }^{7}$. But we formally arrive that the above combined limit in the Euler-Maxwell model still gives the drift diffusion model. In this present paper, our result will show that physically the relaxation regime plays a decisive role in the combined limit. Without loss of generality, we assume $\epsilon=1$. For simplifying the notation, we assume that $\tau=\gamma$. To analyse the combined nonrelativistic relaxation-time limits, we define the scaled variables as in Ref. 16:

$$
(x, t) \rightarrow\left(x, \frac{t}{\tau}\right) .
$$

In this case, the Euler-Maxwell system (1)-(4) is rewritten as:

$$
\left\{\begin{array}{l}
\tau \partial_{t} n+\operatorname{div}(n u)=0, \\
\tau \partial_{t}(n u)+\operatorname{div}(n u \otimes u)+\nabla p(n) \\
\quad=-n E-\gamma n u \times B-\frac{n u}{\tau}, \\
\gamma \tau \partial_{t} E-\nabla \times B=\gamma n u, \\
\operatorname{div} E=b(x)-n, \\
\gamma \tau \partial_{t} B+\nabla \times E=0, \quad \operatorname{div} B=0 .
\end{array}\right.
$$

From the second equation in (8), we can get, using the Maxwell iteration

$$
\begin{aligned}
n u= & -\tau \nabla p(n)-\tau n(E+\gamma u \times B) \\
& -\tau \operatorname{div}(n u \otimes u)-\tau^{2} \partial_{t}(n u) \\
= & -\tau \nabla p(n)-\tau n E+O\left(\tau^{2}\right) .
\end{aligned}
$$

Substituting (9) into the first equation of (8), and taking the formal limit as $\tau \rightarrow 0$ and $\gamma \rightarrow 0$ simultaneously, we obtain the well-known one-fluid drift-diffusion model for semiconductors or plasma

$$
\left\{\begin{array}{l}
\partial_{t} \mathcal{N}=\Delta p(\mathcal{N})+\operatorname{div}(\mathcal{N} \mathcal{E}) \\
\nabla \times \mathcal{E}=0, \operatorname{div} \mathcal{E}=b(x)-\mathcal{N}
\end{array}\right.
$$

and the linear curl-div equation as follows

$$
\nabla \times \mathcal{B}=0, \operatorname{div} \mathcal{B}=0 .
$$

This is because the equation $\nabla \times \mathcal{E}=0$ implies that the electric field is the gradient of some potential function, i.e., $E=\nabla \phi$. So we can obtain $\mathcal{E}=$ $\nabla \Delta^{-1}(b(x)-\mathcal{N})$ from equation $\operatorname{div} \mathcal{E}=b(x)-\mathcal{N}$. Here, the operator $\nabla \Delta^{-1}$ is the mapping from $L\left(\mathcal{T}^{3}\right)$ into $L\left(\mathcal{T}^{3}\right)$. Thus equations (10) is a parabolic-elliptic system, since $p(\mathcal{N})$ is strictly increasing. For the curldiv equations (11), we can take $\mathcal{B}=0$ in the class $\mathbf{m}(\mathcal{B})=\int_{\mathcal{T}^{3}} \mathcal{B} \mathrm{d} x=0$.

We assume that the drift-diffusion model (10) and the curl-div equations (11) have a smooth solution $(\mathcal{N}, \mathcal{E}, \mathcal{B})$ with initial data

$$
(\mathcal{N}(x, 0), \mathcal{E}(x, 0), \mathcal{B}(x, 0))=\left(n_{0}(x), E_{0}(x), 0\right) .
$$

Then, we construct a formal approximation

$$
\left(n_{\tau}, u_{\tau}, E_{\tau}, B_{\tau}\right)=(\mathcal{N},-\tau \mathcal{E}-\tau \nabla h(\mathcal{N}), \mathcal{E}, \mathcal{B})
$$

for the solution $\left(n^{\tau}, u^{\tau}, E^{\tau}, B^{\tau}\right)$ of (8) with initial data

$$
\left.\left(n^{\tau}, u^{\tau}\right)\right|_{t=0}=\left(n_{0}(x),-E_{0}(x)-\nabla h\left(n_{0}\right)\right),
$$

where $E_{0}$ satisfies the following compatibility condition:

$$
\operatorname{div} E_{0}=b(x)-n_{0} .
$$

Then we use energy methods to prove that $\left(n^{\tau}, u^{\tau}, E^{\tau}, B^{\tau}\right)$ exists in the finite time interval and can be expressed as

$$
\left(n^{\tau}, u^{\tau}, E^{\tau}, B^{\tau}\right)=\left(n_{\tau}, u_{\tau}, E_{\tau}, B_{\tau}\right)+O\left(\tau^{2}\right)
$$

in the Sobolev space $H^{s}\left(\mathbb{T}^{3}\right)$.

However, due to the adjunction of non-relativistic regime, the proof to rigid justification becomes more complex and difficult. Because of this effect, some key estimates in Ref. 15 have to be reconsidered, and our analysis depends heavily on the special structure of the Maxwell part of compressible Euler-Maxwell system. 


\section{FORMAL APPROXIMATIONS}

In this section, we give a construction of the approximation $\left(n_{\tau}, u_{\tau}, E_{\tau}, B_{\tau}\right)$ in the convergence assumption for the Euler-Maxwell system $(8)$. Let $(\mathcal{N}, \mathcal{E})$ solve the IVP of the unipolar drift-diffusion model (10). Inspired by the Maxwell iteration described in the introduction, we construct a formal approximation solution:

$$
\left(n_{\tau}, u_{\tau}\right)=(\mathcal{N},-\tau \mathcal{E}-\tau \nabla h(\mathcal{N})) .
$$

It is easy to show that the approximation solution satisfies the following equations

$$
\left\{\begin{array}{l}
\tau \partial_{t} n_{\tau}+u_{\tau} \cdot \nabla n_{\tau}+n_{\tau} \operatorname{div} u_{\tau}=0 \\
\tau \partial_{t} u_{\tau}+\left(u_{\tau} \cdot \nabla\right) u_{\tau}+\nabla h\left(n_{\tau}\right) \\
\quad=-E_{\tau}-\frac{u_{\tau}}{\tau}+\tau^{2} \mathcal{S} \\
\tau \partial_{t} E_{\tau}=n_{\tau} u_{\tau} \\
B_{\tau}=0
\end{array}\right.
$$

where the residue

$$
\begin{aligned}
\mathcal{S}= & \frac{\tau \partial_{t} u_{\tau}+\left(u_{\tau} \cdot \nabla\right) u_{\tau}}{\tau^{2}} \\
= & -\partial_{t}(\mathcal{E}+\nabla h(\mathcal{N})) \\
& +((\mathcal{E}+\nabla h(\mathcal{N})) \cdot \nabla)(\mathcal{E}+\nabla h(\mathcal{N}))
\end{aligned}
$$

Regarding $\left(n_{\tau}, u_{\tau}, E_{\tau}, B_{\tau}\right)$, we have the following regularity result which is based on the well-known calculus inequalities in Sobolev spaces ${ }^{17}$.

Lemma 1 Let $s \geqslant 3$ be an integer. Assume that $h(n) \in C^{\infty}(0, \infty)$ satisfies $h(n)>0$. If

$$
n \in C\left(\left[0, T_{1}\right], H^{s}\right) \cap C^{1}\left(\left[0, T_{1}\right], H^{s-1}\right)
$$

has a positive lower bound, then so does $h=h(n)$. Moreover, if $b(x) \in H^{s-1}$, then

$$
\begin{gathered}
u_{\tau} \in C\left(\left[0, T_{\star}\right], H^{s-1}\right) \cap C^{1}\left(\left[0, T_{1}\right], H^{s-2}\right), \\
E_{\tau} \in C\left(\left[0, T_{1}\right], H^{s}\right) \cap C^{1}\left(\left[0, T_{1}\right], H^{s-1}\right)
\end{gathered}
$$

and

$$
\mathcal{S} \in C\left(\left[0, T_{1}\right], H^{s-1}\right) .
$$

\section{DERIVATION OF ERROR EQUATIONS AND LOCAL EXISTENCE}

Let $\left(n^{\tau}, u^{\tau}, E^{\tau}, B^{\tau}\right)$ be the unknown solution to the problem (8) with the initial data

$$
\begin{aligned}
& \left(n^{\tau}, u^{\tau}, E^{\tau}, B^{\tau}\right)(x, 0) \\
& \quad=\left(n_{0}(x),-\tau E_{0}-\tau \nabla\left(h\left(n_{0}\right)\right), E_{0}, 0\right)
\end{aligned}
$$

and denote by

$\left(N^{\tau}, U^{\tau}, F^{\tau}, G^{\tau}\right)=\left(n^{\tau}-n_{\tau}, u^{\tau}-u_{\tau}, E^{\tau}-E_{\tau}, B^{\tau}\right)$, then, from equations (8) and equations (10), it follows that the error $\left(N^{\tau}, U^{\tau}, F^{\tau}, G^{\tau}\right)$ satisfies

$$
\left\{\begin{array}{c}
\tau \partial_{t} N^{\tau}+u^{\tau} \cdot \nabla N^{\tau}+n^{\tau} \operatorname{div} U^{\tau} \\
=-\left(U^{\tau} \cdot \nabla n_{\tau}+N^{\tau} \operatorname{div} u_{\tau}\right) \\
\tau \partial_{t} U^{\tau}+\left(u^{\tau} \cdot \nabla\right) U^{\tau}+\left(U^{\tau} \cdot \nabla\right) u_{\tau} \\
\quad+h^{\prime}\left(n^{\tau}\right) \nabla N^{\tau} \\
\quad+\left(h^{\prime}\left(n^{\tau}\right)-h^{\prime}\left(n_{\tau}\right)\right) \nabla n_{\tau}+F^{\tau} \\
\quad+\gamma u^{\tau} \times G^{\tau}=-\frac{U^{\tau}}{\tau}-\tau^{2} \mathcal{S} \\
\gamma \tau \partial_{t} F^{\tau}-\nabla \times G^{\tau}=\gamma\left(n^{\tau} U^{\tau}+N^{\tau} u_{\tau}\right), \\
\gamma \tau \partial_{t} G^{\tau}+\nabla \times F^{\tau}=0 \\
\left.V^{\tau}\right|_{t=0}=V_{0}^{\tau}
\end{array}\right.
$$

where, $n^{\tau}=N^{\tau}+n_{\tau}$ and $u^{\tau}=U^{\tau}+u_{\tau}$.

Set

$$
V^{\tau}=\left(\begin{array}{c}
N^{\tau} \\
U^{\tau} \\
F^{\tau} \\
G^{\tau}
\end{array}\right), V_{0}^{\tau}=\left(\begin{array}{c}
N_{0}^{\tau} \\
U_{0}^{\tau} \\
F_{0}^{\tau} \\
G_{0}^{\tau}
\end{array}\right)
$$

$$
\begin{gathered}
D_{0}^{\tau}=\left(\begin{array}{cc}
\tau I_{4 \times 4} & 0 \\
0 & \gamma \tau I_{6 \times 6}
\end{array}\right) \\
A_{j}\left(V^{\tau}\right)=\left(\begin{array}{cccc}
\left(u^{\tau}\right)_{j} & n^{\tau} e_{j}^{T} & 0 & 0 \\
h^{\prime}\left(n^{\tau}\right) e_{j} & \left(u^{\tau}\right)_{j} I_{3 \times 3} & 0 & 0 \\
0 & 0 & 0 & C_{j} \\
0 & 0 & C_{j}^{T} & 0
\end{array}\right),
\end{gathered}
$$

$$
\begin{aligned}
& H_{1}\left(V^{\tau}\right) \\
& =\left(\begin{array}{c}
U^{\tau} \cdot \nabla n_{\tau}+N^{\tau} \operatorname{div} u_{\tau} \\
\left(U^{\tau} \cdot \nabla\right) u_{\tau}+\left(h^{\prime}\left(n^{\tau}\right)-h^{\prime}\left(n_{\tau}\right)\right) \nabla n_{\tau} \\
0 \\
0
\end{array}\right)
\end{aligned}
$$

$$
H_{2}\left(V^{\tau}\right)=\left(\begin{array}{c}
0 \\
F^{\tau} \\
0 \\
0
\end{array}\right)
$$

$$
H_{3}\left(V^{\tau}\right)=\left(\begin{array}{c}
0 \\
-u^{\tau} \times G^{\tau} \\
n^{\tau} U^{\tau}+N^{\tau} u_{\tau} \\
0
\end{array}\right)
$$

and

$$
H_{4}\left(V^{\tau}\right)=\left(\begin{array}{c}
0 \\
U^{\tau} \\
0 \\
0
\end{array}\right), H_{5}\left(V^{\tau}\right)=\left(\begin{array}{l}
0 \\
\mathcal{S} \\
0 \\
0
\end{array}\right),
$$

where $\left(e_{1}, e_{2}, e_{3}\right)$ is the canonical base of $\mathbb{R}^{3}$, $I_{d \times d}(d=3,4,6)$ is a $d$ order unit matrix, $y_{i}$ denotes 
the $i$ th component of $y \in \mathbb{R}^{3}$. each $C_{j}(j=1,2,3)$ is a constant skew matrix, specifically

$$
\begin{gathered}
C_{1}=\left(\begin{array}{ccc}
0 & 0 & 0 \\
0 & 0 & -1 \\
0 & 1 & 0
\end{array}\right), \quad C_{2}=\left(\begin{array}{ccc}
0 & 0 & -1 \\
0 & 0 & 0 \\
1 & 0 & 0
\end{array}\right), \\
C_{3}=\left(\begin{array}{ccc}
0 & 1 & 0 \\
-1 & 0 & 0 \\
0 & 0 & 0
\end{array}\right) .
\end{gathered}
$$

Thus problem (21) for the unknown $V^{\tau}$ can be rewritten as

$$
\left\{\begin{array}{c}
D_{0}^{\tau} \partial_{t} V^{\tau}+\sum_{i=1}^{3} A_{j}\left(V^{\tau}\right) \partial_{x_{j}} V^{\tau} \\
=-H_{1}\left(V^{\tau}\right)-H_{2}\left(V^{\tau}\right)+\gamma H_{3}\left(V^{\tau}\right) \\
-\frac{1}{\tau} H_{4}\left(V^{\tau}\right)-\tau^{2} H_{5}\left(V^{\tau}\right), \\
\left.V^{\tau}\right|_{t=0}=V_{0}^{\tau} .
\end{array}\right.
$$

It is not difficult to see that the equations of $V^{\tau}$ in (21) are symmetrizable hyperbolic, i.e., if we introduce

$$
A_{0}\left(N^{\tau}\right)=\left(\begin{array}{ccc}
h^{\prime}\left(n^{\tau}\right) & 0 & 0 \\
0 & n^{\tau} I_{3 \times 3} & 0 \\
0 & 0 & I_{6 \times 6}
\end{array}\right)
$$

which is positively definite when $N^{\tau}+n_{\tau} \geqslant \widetilde{C_{0}}>0$ for $0<\tau \leqslant \tau_{0} \ll 1$. Thus by the classical existence theory of the local smooth solutions of the symmetrizable hyperbolic system, see Ref. 17, we have

Proposition 1 Let $M>0$ and $V_{0}^{\tau}$ satisfy $V_{0}^{\tau} \in$ $\left(H^{s}\left(\mathcal{T}^{3}\right)\right), s \geqslant 3$ and $\left\|N_{0}^{\tau}\right\|_{H^{s}\left(\mathcal{T}^{3}\right)} \leqslant \lambda$ for any given $\lambda>0$ (to be chosen sufficiently small so that $M \lambda C_{s} \leqslant \frac{1}{2}$, where $C_{s}$ is Sobolev's embedding constant). Then for any fixed $\tau$ or $\gamma$ there is a maximal existence time $0<T_{\tau} \leqslant \infty$ and a unique smooth solution $V^{\tau} \in \bigcap_{l=0}^{1} C^{l}\left(\left[0, T_{\tau}\right) ; H^{s-l}\left(\mathcal{T}^{3}\right)\right)$ to the error system (21), well-defined on the interval $\left[0, T_{\tau}\right)$. Moreover, if $T_{\tau}<\infty$ and there exists a positive constant $M$ satisfying $C_{s} M \lambda<\frac{1}{2}$ such that

$$
\sup _{0 \leqslant t \leqslant T_{\tau^{-}}^{-}}\left\|N^{\tau}(t)\right\|_{H^{s}\left(\mathcal{T}^{3}\right)}=M \lambda
$$

holds, then one gets either

$$
\lim _{t \rightarrow T_{\tau}^{-}}\left\|N^{\tau}(t)\right\|_{H^{s}\left(\mathcal{T}^{3}\right)}=M \lambda
$$

or

$$
\lim _{t \rightarrow T_{\tau}^{-}}\left\|\left(U^{\tau}(t), F^{\tau}(t), G^{\tau}(t)\right)\right\|_{H^{s}\left(\mathcal{T}^{3}\right)}=\infty .
$$

Noting that the constant matrix $D_{0}^{\tau}$ does not depend upon $\tau$ in the way of $A_{0}\left(C+\tau V^{\tau}\right)$, where $C$ is the constant vector. Hence the singular limit theory for symmetrizable hyperbolic systems developed by Klainerman and Majda ${ }^{18}$ or extended further by Schochet ${ }^{19}$ cannot be applied here to obtain the uniformly a priori estimates of the solution $V^{\tau}$ with respect to $\tau$. This assumption is obviously not satisfied by our present system (8). Usually it is difficult to establish uniformly a priori estimates on the error $F^{\tau}$ of the electric field with respect to due to the singular structure of the matrix $D_{0}^{\tau}$.

Now we begin to justify the convergence of compressible Euler-Maxwell equations to drift-diffusion model when $\gamma \rightarrow 0$ and $\tau \rightarrow 0$ simultaneity. To this end, by the local existence theory and extension method, it suffices to obtain the uniform estimates of the smooth solutions to (21) with respect to the parameter $\tau$ so as to guarantee $T_{\tau} \geqslant T_{0}$ for any given $T_{0}<T_{1}$.

In the following, the repeated index for the sum, and $(\cdot, \cdot)$ stands for the $L^{2}$ inner product of the two scalar or vector functions in $\mathcal{T}^{3}$. Also, we denote

$$
\begin{gathered}
\|\cdot\|=\|\cdot\|_{L^{2}\left(\mathcal{T}^{3}\right)}, \quad\|\cdot\|_{s}=\|\cdot\|_{H^{s}\left(\mathcal{T}^{3}\right)}, \quad s \in \mathbb{N}^{*}, \\
\left\|\left|V^{\tau}\left\|\left.\right|_{0, T}=\sup _{0 \leqslant t \leqslant T}\right\| V^{\tau}(t) \|,\right.\right. \\
\left\|V^{\tau}\right\|\left\|_{s, T}=\sup _{0 \leqslant t \leqslant T}\right\| \mid V^{\tau}(t)\|\|_{s}, \quad s \in \mathbb{N}^{*},
\end{gathered}
$$

where $V^{\tau}=\left(N^{\tau}, U^{\tau}, F^{\tau}, G^{\tau}\right)^{T}$.

Let $T_{1}$ be the maximal existence time of the solution given in Lemma 1. In the following, assuming the conditions of Lemma 1, we establish a priori estimates by the elaborate energy methods in several steps.

Denote by $T=T^{\tau}=\min \left\{T_{1}, T_{\tau}\right\}$, and by $C>$ 0 a constant which depends upon $T$ and but does not depend upon $M, T$, and $\tau$.

\section{THE MAIN RESULT}

Having constructed the formal approximation $\left(n_{\tau}, u_{\tau}, E_{\tau}, B_{\tau}\right)$ for the periodic IVP of the compressible Euler-Maxwell system (8), we prove here the validity of the approximation under some regularity assumptions on the given data and an existence result for the IVP. The main result of this paper is stated as follows.

Theorem 1 Let $s \geqslant 3, s \in \mathbb{N}$. Assume $p \in$ $C^{\infty}(0, \infty), p^{\prime}(n)>0, b(x) \in H^{s}\left(\mathcal{T}^{3}\right)$ and that the drift-diffusion model (10) has a solution $\left(n_{\tau}, E_{\tau}\right) \in$ $C\left(\left[0, T_{1}\right], H^{s+2}\right) \cap C^{1}\left(\left[0, T_{1}\right], H^{s+1}\right)$ with $n$ being bounded away from zero. Then, for sufficiently small 
$0<\tau<\tau_{0} \ll 1$, the Euler-Maxwell equations (8) with periodic initial data (19) has a unique solution $\left(n^{\tau}, u^{\tau}, E^{\tau}, B^{\tau}\right)$ satisfying $\left(n^{\tau}, u^{\tau}, E^{\tau}, B^{\tau}\right) \in$ $C\left([0, T], H^{s}\right)$, and there exists a constant $C_{0}>0$, independent of $\tau$ but dependent on $T<\infty$, such that $\left|\left\|\left(n^{\tau}-n_{\tau}, u^{\tau}-u_{\tau}, E^{\tau}-E_{\tau}, B^{\tau}\right)(t) \mid\right\|_{s, T} \leqslant C_{0} \tau\right.$.

Proof: From the equations in (22) it follows that the error satisfies

$$
\begin{aligned}
\partial_{t} V^{\tau}+ & \left(D_{0}^{\tau}\right)^{-1} \sum_{j=1}^{3} A_{j}\left(V^{\tau}\right) \partial_{x_{j}} V^{\tau} \\
& =\left(D_{0}^{\tau}\right)^{-1} \sum_{i=1}^{5} \mathcal{H}_{i},
\end{aligned}
$$

where $\mathcal{H}_{i}(i=1,2,3,4,5)$ is defined as follows

$$
\begin{aligned}
\left(\mathcal{H}_{1},\right. & \left.-\mathcal{H}_{2}, \mathcal{H}_{3}, \mathcal{H}_{4}, \mathcal{H}_{5}\right) \\
& =\left(-H_{1}, H_{2}, \gamma H_{3},-\frac{1}{\tau} H_{4},-\tau^{2} H_{5}\right)
\end{aligned}
$$

Let $\alpha \in \mathbb{N}^{3}$ be a multi-index with $|\alpha| \leqslant s$ with $s \geqslant 3$. We differentiate this equation with $\partial_{x}^{\alpha}$ to get

$$
\begin{aligned}
& \partial_{t} \partial_{x}^{\alpha} V^{\tau}+\left(D_{0}^{\tau}\right)^{-1} \sum_{j=1}^{3} A_{j}\left(V^{\tau}\right) \partial_{x_{j}} \partial_{x}^{\alpha} V^{\tau} \\
& =\left(D_{0}^{\tau}\right)^{-1} \sum_{i=1}^{6} \partial_{x}^{\alpha} \mathcal{H}_{i}
\end{aligned}
$$

where

$$
\mathcal{H}_{6}=\sum_{j=1}^{3}\left(A_{j}\left(V^{\tau}\right) \partial_{x_{j}} \partial_{x}^{\alpha} V^{\tau}-\partial_{x}^{\alpha}\left(A_{j}\left(V^{\tau}\right) \partial_{x_{j}} V^{\tau}\right)\right) \text {. }
$$

We now introduce the energy

$$
\mathbb{E}(t)=\left(A_{0}\left(N^{\tau}\right) V^{\tau}, V^{\tau}\right),
$$

where

$$
(u, v)=\int_{\mathcal{T}^{3}} u \cdot v \mathrm{~d} x,\|u\|=\left(\int_{\mathcal{T}^{3}}|u|^{2} \mathrm{~d} x\right)^{\frac{1}{2}} .
$$

Since matrices $A_{0}\left(N^{\tau}\right)$ and $A_{0}\left(N^{\tau}\right) A_{j}\left(V^{\tau}\right)(j=$ $1,2,3)$ are symmetric, we take the $L^{2}$ inner product of equation (25) with $A_{0}\left(N^{\tau}\right) \partial_{x}^{\alpha} V^{\tau}$, one gets

$$
\begin{aligned}
\frac{\mathrm{d}}{\mathrm{d} t} \mathbb{E}(t)= & 2\left(D_{0}^{\tau}\right)^{-1} \sum_{i=1}^{6}\left(A_{0}\left(N^{\tau}\right) \partial_{x}^{\alpha} V^{\tau}, \partial_{x}^{\alpha} \mathcal{H}_{i}\right) \\
& +2\left(\mathcal{Z} \partial_{x}^{\alpha} V^{\tau}, \partial_{x}^{\alpha} V^{\tau}\right) \\
= & \sum_{i=1}^{7} \mathcal{Q}_{i}^{\alpha},
\end{aligned}
$$

where

$$
\mathcal{Z}=\partial_{t} A_{0}\left(N^{\tau}\right)+\left(D_{0}^{\tau}\right)^{-1} \sum_{j=1}^{3} \partial_{x_{j}}\left(A_{0}\left(N^{\tau}\right) A_{j}\left(V^{\tau}\right)\right) .
$$

Note that for any $\omega \in \mathbb{R}^{6 \times 1},(\omega, \Phi \omega)=0$,where

$$
\Phi=\left(\begin{array}{cc}
0 & C_{j} \\
C_{j}^{T} & 0
\end{array}\right)
$$

is a skew matrix. By a direct calculation, we get

$$
\begin{aligned}
& \mathcal{Q}_{1}^{\alpha} \\
&=-\frac{2}{\tau} \int_{\mathcal{T}^{3}} h^{\prime}\left(n^{\tau}\right) \partial_{x}^{\alpha} N^{\tau} \partial_{x}^{\alpha} \mathcal{F}_{1} \mathrm{~d} x \\
&-\frac{2}{\tau} \int_{\mathcal{T}^{3}}\left(n^{\tau}\right) \partial_{x}^{\alpha} U^{\tau} \cdot \partial_{x}^{\alpha}\left(\left(U^{\tau} \cdot \nabla\right) u_{\tau}\right) \mathrm{d} x \\
&-\frac{2}{\tau} \int_{\mathcal{T}^{3}} n^{\tau} \partial_{x}^{\alpha} U^{\tau} \cdot \partial_{x}^{\alpha}\left(\mathcal{F}_{2} \nabla n_{\tau}\right) \mathrm{d} x \\
& \mathcal{Q}_{2}^{\alpha}=-\frac{2}{\tau} \int_{\mathcal{T}^{3}} n^{\tau} \partial_{x}^{\alpha} U^{\tau} \cdot \partial_{x}^{\alpha} F^{\tau} \mathrm{d} x \\
& \mathcal{Q}_{3}^{\alpha}=-\frac{2}{\tau} \int_{\mathcal{T}^{3}} n^{\tau} \partial_{x}^{\alpha} U^{\tau} \cdot \partial_{x}^{\alpha}\left(\left(U^{\tau}+u_{\tau}\right) \times G^{\tau}\right) \mathrm{d} x \\
&\left.+\frac{2}{\tau} \int_{\mathcal{T}^{3}} \partial_{x}^{\alpha} n^{\tau} U^{\tau}+N^{\tau} u_{\tau}\right) \cdot \partial_{x}^{\alpha} F^{\tau} \mathrm{d} x \\
& \mathcal{Q}_{4}^{\alpha}=-\frac{2}{\tau^{2}} \int_{\mathcal{T}^{3}} n^{\tau}\left|\partial_{x}^{\alpha} U^{\tau}\right|^{2} \mathrm{~d} x \\
& \mathcal{Q}_{5}^{\alpha}=-\tau \int_{\mathcal{T}^{3}} n^{\tau} \partial_{x}^{\alpha} U^{\tau} \cdot \partial_{x}^{\alpha} \mathcal{S} \mathrm{d} x \\
& \mathcal{Q}_{6}^{\alpha}= \frac{2}{\tau} \int_{\mathcal{T}^{3}} h^{\prime}\left(n^{\tau}\right) \partial_{x}^{\alpha} N^{\tau} \mathcal{F}_{3} \mathrm{~d} x \\
&+\frac{2}{\tau} \int_{\mathcal{T}^{3}} n^{\tau} \partial_{x}^{\alpha} U^{\tau} \mathcal{F}_{4} \mathrm{~d} x \\
& \mathcal{Q}_{7}^{\alpha}= 2 \int_{\mathcal{T}^{3}}\left(\partial_{t} h^{\prime}\left(n^{\tau}\right)+\frac{1}{\tau} u^{\tau} \cdot \nabla h^{\prime}\left(n^{\tau}\right)\right. \\
&\left.+\frac{1}{\tau} h^{\prime}\left(n^{\tau}\right) \operatorname{div} u^{\tau}\left|\partial_{x}^{\alpha} N^{\tau}\right|^{2}\right) \mathrm{d} x \\
&+2 \int_{\mathcal{T}^{3}}\left(\partial_{t} n^{\tau}+\frac{1}{\tau} \operatorname{div}\left(n^{\tau} u^{\tau}\right)\left|\partial_{x}^{\alpha} U^{\tau}\right|^{2} \mathrm{~d} x\right. \\
& \mathcal{F}_{5}\left|\partial_{x}^{\alpha} N^{\tau}\right|^{2} \mathrm{~d} x \\
&=
\end{aligned}
$$

where

$$
\begin{aligned}
\mathcal{F}_{1}= & U^{\tau} \cdot \nabla n_{\tau}+N^{\tau} \operatorname{div} u_{\tau}, \\
\mathcal{F}_{2}= & h^{\prime}\left(n^{\tau}\right)-h^{\prime}\left(n_{\tau}\right), \\
\mathcal{F}_{3}= & u^{\tau} \nabla \partial_{x}^{\alpha} N^{\tau}-\partial_{x}^{\alpha}\left(u^{\tau} \nabla N^{\tau}\right) \\
& +n^{\tau} \operatorname{div} \partial_{x}^{\alpha} U^{\tau}-\partial_{x}^{\alpha}\left(n^{\tau} \operatorname{div} U^{\tau}\right), \\
\mathcal{F}_{4}= & h^{\prime}\left(n^{\tau}\right) \nabla \partial_{x}^{\alpha} N^{\tau}-\partial_{x}^{\alpha}\left(h^{\prime}\left(n^{\tau}\right) \nabla N^{\tau}\right) \\
& +\left(u^{\tau} \cdot \nabla\right) \partial_{x}^{\alpha} U^{\tau}-\partial_{x}^{\alpha}\left(\left(u^{\tau} \cdot \nabla\right) U^{\tau}\right), \\
\mathcal{F}_{5}= & \left(h^{\prime}\left(n^{\tau}\right)-h^{\prime \prime}\left(n^{\tau}\right) n^{\tau}\right) \operatorname{div} u^{\tau} .
\end{aligned}
$$


Let

$$
\mathcal{D}=\mathcal{D}(t)=\frac{1}{\tau}\left\|V(\cdot, t)^{\tau},\right\|_{s} .
$$

For $s>s_{0} \geqslant 3$, we use equation (16) and the wellknown embedding inequality to obtain

$$
\begin{aligned}
\left|\operatorname{div}\left(U^{\tau}+u_{\tau}\right)\right| & \leqslant C\left\|\operatorname{div} u_{\tau}\right\|_{s_{0}}+C\left\|\operatorname{div} U^{\tau}\right\|_{s_{0}} \\
& \leqslant C \tau(1+\mathcal{D}) .
\end{aligned}
$$

Now, we begin to estimate each $\mathcal{Q}_{i}^{\alpha}(i=1, \ldots, 7)$.

For the $\mathcal{Q}_{i}^{\alpha}(i=1,2,3,5)$ term, we use CauchySchwartz's inequality and Sobolev's lemma to obtain

$$
\begin{aligned}
& \mathcal{Q}_{1}^{\alpha} \leqslant C\left(1+\mathcal{D}^{s}\right)\left\|\left(N^{\tau}, U^{\tau}\right)\right\|_{|\alpha|}^{2}+\frac{\|U\|_{s}^{2}}{2 \widetilde{C_{0}} \tau^{2}} \\
& \mathcal{Q}_{2}^{\alpha} \leqslant C\left(1+\mathcal{D}^{2 s}\right)\left\|F^{\tau}\right\|_{|\alpha|}^{2}+\frac{\|U\|_{s}^{2}}{2 \widetilde{C_{0}} \tau^{2}} \\
& \mathcal{Q}_{3}^{\alpha} \leqslant C\left(1+\mathcal{D}^{2 s}\right)\left\|\left(F^{\tau}, G^{\tau}\right)\right\|_{|\alpha|}^{2}+\frac{\|U\|_{s}^{2}}{2 \widetilde{C_{0}} \tau^{2}} \\
& \mathcal{Q}_{5}^{\alpha} \leqslant C\left(1+\mathcal{D}^{2 s}\right)\left\|U^{\tau}\right\|_{|\alpha|}^{2}+\frac{\|U\|_{s}^{2}}{2 \widetilde{C_{0}} \tau^{2}}+C \tau^{4} .
\end{aligned}
$$

For $\mathcal{Q}_{4}^{\alpha}$, noting that $N^{\tau}+n_{\tau} \geqslant \widetilde{C_{0}}>0$ for $0<$ $\tau \leqslant \tau_{0} \ll 1$, we have

$$
\mathcal{Q}_{4}^{\alpha} \leqslant-\frac{2\|U\|_{s}^{2}}{\widetilde{C_{0}} \tau^{2}}
$$

Using the Moser-type calculus inequalities ${ }^{18,20}$, we obtain the estimation of $\mathcal{Q}_{6}^{\alpha}$

$$
Q_{6}^{\alpha} \leqslant C\left(1+\mathcal{D}^{2 s}\right)\left\|\left(N^{\tau}, U^{\tau}\right)\right\|_{|\alpha|}^{2} .
$$

For $\mathcal{Q}_{7}^{\alpha}$, we estimate it as follows

$$
\begin{aligned}
Q_{7}^{\alpha} & \leqslant C \frac{1}{\tau}\left|h^{\prime}\left(n^{\tau}\right)-h^{\prime \prime}\left(n^{\tau}\right) n\left\|\operatorname{div} u^{\tau} \mid\right\| N_{\alpha}^{\tau} \|^{2}\right. \\
& \leqslant C\left(1+\mathcal{D}^{2 s}\right)\left\|N^{\tau}\right\|_{|\alpha|}^{2} .
\end{aligned}
$$

Substituting the estimates (35)-(41) into equality (26) yields

$$
\frac{\mathrm{d}}{\mathrm{d} t} \mathbb{E}(t) \leqslant C\left(1+\mathcal{D}^{2 s}\right)\left\|\left(N^{\tau}, U^{\tau}, F^{\tau}, G^{\tau}\right)\right\|_{|\alpha|}^{2}+C \tau^{4}
$$

Note that $C^{-1}\left\|\partial_{x}^{\alpha} V^{\tau}\right\|^{2} \leqslant \mathbb{E}(t) \leqslant C\left\|\partial_{x}^{\alpha} V^{\tau}\right\|^{2}$. We integrate (42) in the interval $[0, T]$ to obtain

$$
\begin{aligned}
& \left\|\partial_{x}^{\alpha} V^{\tau}(T)\right\|^{2} \\
\leqslant & C \int_{0}^{T}\left(1+\mathcal{D}^{2 s}\right)\left\|\left(N^{\tau}, U^{\tau}, F^{\tau}, G^{\tau}\right)\right\|_{|\alpha|}^{2} \mathrm{~d} t \\
& +C T \tau^{4} .
\end{aligned}
$$

Here we have used the fact that the initial data are in equilibrium. By summing this over all multi-indexes $|\alpha| \leqslant s$, we get

$$
\begin{aligned}
& \left\|V^{\tau}(T)\right\|_{s}^{2} \\
\leqslant & C \int_{0}^{T}\left(1+\mathcal{D}^{2 s}\right)\left\|\left(N^{\tau}, U^{\tau}, F^{\tau}, G^{\tau}\right)\right\|_{s}^{2} \mathrm{~d} t \\
& +C T \tau^{4} .
\end{aligned}
$$

We apply Gronwall's lemma to (44) to get

$$
\left\|V^{\tau}(T)\right\|_{s}^{2} \leqslant C T \tau^{4} \exp \left(\int_{0}^{T}\left(1+\mathcal{D}^{2 s}\right) \mathrm{d} t\right) .
$$

Since $\left\|V^{\tau}\right\|_{s}=\tau \mathcal{D}$, it follows from (45) that

$$
\|\mathcal{D}(T)\|_{s}^{2} \leqslant C T \tau^{2} \exp \left(\int_{0}^{T}\left(1+\mathcal{D}^{2 s}\right) \mathrm{d} t\right)=\mathcal{W}(T) .
$$

It is easy to get

$\mathcal{W}^{\prime}(t)=C\left(1+\mathcal{D}^{2 s}\right) \mathcal{W}(t) \leqslant C \mathcal{W}(t)+C \mathcal{W}^{s+1}(t)$

Applying Gronwall inequality yields

$$
\mathcal{W}(t) \leqslant \exp (C T)
$$

for $t \in[0, T]$ if we choose $\tau$ so small that

$$
\mathcal{W}(0)=C T \tau^{2}<\exp (-C T) .
$$

Because of (46), there exists a constant $C_{0}$, independent of $\tau$, such that

$$
\mathcal{W}(t)<C_{0}
$$

for any $t \in[0, T]$. Finally, the theorem is concluded from (45) with (47). This completes the proof of Theorem 1.

Acknowledgements: The authors would like to thank the referee for their pertinent comments and advice. This work was supported by the United Fund for Fostering Talents of NSFC-Henan (Grant No. U1204103) and Research Initiation Project for High level Talents (40118) of North China University of Water Resources and Electric Power.

\section{REFERENCES}

1. Chen F (1984) Introduction to Plasma Physics and Controlled Fusion. Vol 1, Plenum Press, New York.

2. Markowich F, Ringhofer C, Schmeiser C (1990) Semiconductor Equations, Springer.

3. Alsunaidi M, El-Ghazaly S (1994) High frequency time domain modeling of GaAs FET's using the hydrodynamic model coupled with Maxwell's equations. In: IEEE Symposium, San Diego, pp 397-400. 
4. Alsunaidi M, Imtiaz S, El-Ghazaly S (1996) Electromagnetic wave effects on microwave transistors using a full-wave time-domain model. IEEE Trans Microw Theor Tech 44, 799-808.

5. Chen G, Jerome J, Wang D (2000) Compressible EulerMaxwell equations. Transport Theor Stat Phys 29, 311-31.

6. Jerome J (2003) The Cauchy problem for compressible hydrodynamic-Maxwell systems: A local theory for smooth solutions. Differ Integr Equat 16, 1345-68.

7. Peng Y, Wang S (2007) Convergence of compressible Euler-Maxwell equations to compressible EulerPoisson equations. Chin Ann Math 28(B), 583-602.

8. Yang J, Wang S (2009) The non-relativistic limit of Euler-Maxwell equations for two-fluid plasma. Nonlinear Anal Theor Meth Appl 72, 1829-40.

9. Yang J, Wang S (2009) Convergence of the nonisentropic Euler-Maxwell equations to compressible EulerPoisson equations. J Math Phys 50, 123508.

10. Peng Y, Wang S (2008) Convergence of compressible Euler-Maxwell equations to incompressible Euler equations. Comm Part Differ Equat 33, 349-476.

11. JF, Coulombel J, Goudon T (2007) The strong relaxation limit of the multidimensional isothermal Euler equations. Trans Am Math Soc 359, 637-48.

12. Xu J (2010) Strong relaxation limit of multidimensional isentropic Euler equations. $Z$ Angew Math Phys 61, 389-400.

13. Yong W (2004) Diffusive relaxation limit of multidimensional isentropic hydrodynamic models for semiconductors, SIAM J Appl Math 64, 1737-48.

14. Li Y (2007) Relaxation time limits problem for hydrodynamics models in semiconductor science. Acta Mathematica Scientia, 27B, 437-48.

15. Yang J, Wang S, Zhao J (2011) The relaxation-time limit in the compressible Euler-Maxwell equations. Nonlin Anal 74, 7005-11.

16. Marcati P, Natalini R (1995) Weak solutions to a hydrodynamic model for semiconductors and relaxation to the drift-diffusion equations. Arch Ration Mech Anal 129, 129-45.

17. Majda A (1984) Compressible Fluid Flow and Systems of Conservation Laws in Several Space Variables. Springer-Verlag, New-York.

18. Klainerman S, Majda A (1981) Singular limits of quasilinear hyperbolic systems with large parameters and the incompressible limit of compressible fluids. Comm Pure Appl Math 34, pp. 481-524.

19. Schochet S (1994) Fast singular limits of hyperbolic PDEs. J Differ Equat 114, 476-512.

20. Klainerman S, Majda A (1982) Compressible and incompressible fluids. Comm Pure Appl Math 35, 629-51. 